OU-TAP-241

TU-738

hep-ph/0502211

\title{
Neutralino Dark Matter from Heavy Gravitino Decay
}

\author{
Kazunori Kohri ${ }^{1,2}$, Masahiro Yamaguchi ${ }^{3}$, and Jun'ichi Yokoyama ${ }^{2}$ \\ ${ }^{1}$ Harvard-Smithsonian Center for Astrophysics, 60 Garden Street, Cambridge, MA 02138, USA \\ ${ }^{2}$ Department of Earth and Space Science, Graduate School of Science, \\ Osaka University, Toyonaka 560-0043, Japan \\ ${ }^{3}$ Department of Physics, Tohoku University, Sendai 980-8578, Japan
}

(March 15, 2018)

\begin{abstract}
We propose a new scenario of non-thermal production of neutralino cold dark matter, in which the overproduction problem of lightest supersymmetric particles (LSPs) in the standard thermal history is naturally solved. The mechanism requires a heavy modulus field which decays mainly to ordinary particles releasing large entropy to dilute gravitinos produced just after inflation and thermal relics of LSPs. Significant amount of gravitinos are also pair-produced at the decay, which subsequently decay into the neutralinos. We identify the regions of the parameter space in which the requisite abundance of the neutralino dark matter is obtained without spoiling the big-bang nucleosynthesis by injection of hadronic showers from gravitino decay. The neutralino abundance obtained in this mechanism is insensitive to the details of the superparticle mass spectrum, unlike the standard thermal abundance. We also briefly mention the testability of the scenario in future experiments.
\end{abstract}




\section{INTRODUCTION}

Supersymmetry (SUSY) has been a promising candidate for new physics beyond the standard model, not only because it is an attractive solution to the naturalness problem associated with the electroweak scale, but also because it provides a natural candidate for cold dark matter. Indeed, the relic abundance of the lightest supersymmetric particle (LSP), which is stable by virtue of the $\mathrm{R}$ parity conservation, may naturally fall into the critical density with plausible values of its mass and couplings under the standard thermal history of the early universe. This concordance was regarded as one of the triumph of particle cosmology in the last century.

Recent progress on the determination of the the cold dark matter (CDM) abundance, however, has cast a shadow on the above simplistic view of the standard SUSY cosmology. In particular, the value indicated by the WMAP measurement pushes the preferred regions of the SUSY parameter to only tiny and special corners of the parameter space. In fact the WMAP data implies the Dark Matter abundance [1]

$$
\Omega_{\mathrm{CDM}} h^{2}=0.1126_{-0.0181}^{+0.0161}
$$

at $95 \%$ C.L., where $\Omega_{\mathrm{CDM}}$ represents the current value of the density parameter of the CDM component, and $h$ is the Hubble parameter in unit of $100 \mathrm{~km} / \mathrm{s} / \mathrm{Mpc}$. This is much smaller than unity. On the other hand, assuming the standard thermal history of the universe, the thermal relic abundance of the neutralinos can be computed in a standard manner. Importantly it is known that in generic regions of the SUSY parameter space, the relic abundance computed in this way tends to exceed the value the WMAP suggests [2]. The right amount of the neutralino abundance is obtained only in tiny and special regions of the parameter space where some enhancement mechanisms of neutralino annihilation take place. They include 1) light sfermions, 2) coannihilation with e.g. chargino, stau, or stop, 3) resonance enhancement, and 4) annihilation into $W$ pair.

Furthermore this tension we now have may become real conflict if the LHC experiments, for instance, will find SUSY particles outside the regions where the calculated relic abundance is consistent with that implied by the WMAP measurement.

At this point, one should recognize that the argument of the thermal relic abundance strongly relies on the standard thermal history of the universe. The fact that the enhanced annihilation of neutralinos is required to meet observation may imply that the universe might have evolved in a non-standard manner. Alternatively, we may find a natural solution to the dark matter problem making use of a non-standard thermal history without any enhancement of the annihilation processes, which is the central topic of the present paper.

Tension in the SUSY cosmology with the standard thermal history increases even more when one turns to the recent reanalysis of the gravitino problem which includes the effects of hadronic showers on the abundance of the light elements produced by big-bang nucleosynthesis (BBN) [3]. It is well-known that decays of long-lived particles such as gravitinos would spoil the success of the big-bang nucleosynthesis because of the production and destruction of the light elements by emitted high-energy photons or hadrons [4-8]. If the gravitino mass is just around the weak scale, very severe upper bounds come from the overproduction of the ${ }^{6} \mathrm{Li}$ abundance and the D abundance. We should also stress that inclusion of the hadronic shower also put a rather stringent bound for heavier gravitino whose lifetime is even shorter 
than $1 \mathrm{sec}$ This is because the hadronic shower produces pions and nucleons which interact with ambient nucleons in the thermal bath, changing the neutron to proton ratio and hence the ${ }^{4} \mathrm{He}$ abundance $[5,9]$. Note that the argument is based on the standard thermal history of the universe in which there is no dilution or extra production of gravitinos after reheating epoch of primordial inflation $[10,11]$.

It is, however, quite plausible that the universe did not follow the standard thermal history described above. This is particularly the case when there are some moduli fields whose masses are not far away from the electroweak scale. The coherent oscillation of the moduli fields is likely to dominate the energy density of the universe at some epoch in the early universe, followed by rather late decays [12]. If the mass is in the electroweak scale, the lifetime of the moduli fields is much longer than $1 \mathrm{sec}$, meaning that the entropy production at the moduli decays takes place with reheat temperature lower than $1 \mathrm{MeV}$. This would upset the success of the BBN. The disaster can be avoided if the moduli masses are somewhat larger than the electroweak scale [14-17].

In a previous paper [13], we have shown that the gravitino abundance can be regulated to an observationally acceptable level if we take entropy production due to moduli decay into account. In fact we have shown that it is possible to satisfy the severe constraints imposed by its hadronic decay [3] even when the reheat temperature after inflation was as high as $\sim 10^{16} \mathrm{GeV}$ and the new production mechanism of gravitinos from moduli decay is taken into account [13].

In this paper, we would like to explore this line of argument and propose a new scenario in which the neutralino dark matter is produced in a non-thermal way. In our scenario, the neutralinos of the thermal origin are all diluted by the entropy production at the moduli decay. The dark matter neutralinos are produced, however, by the decay of gravitinos, following the moduli decay to gravitinos.

It is very non-trivial whether this mechanism survives severe constraints from BBN on the gravitino decay which produces hadronic and/or electromagnetic showers. We will show that in a range of the mass parameters, the gravitino decay survives the constraint from the BBN, whereas the abundance of the neutralino LSPs is in accord with the cosmological observations. Thus our model offers a consistent scenario of cosmic evolution in the new era of precision cosmology where the cold dark matter abundance is much smaller than previously expected and the gravitino problem is much more aggravated. We will also see that the scenario requires small hierarchy among moduli, gravitino and soft SUSY breaking masses. We will argue how this small hierarchy is naturally realized.

The organization of the paper is as follows. In the next section we outline the basic features of our scenario. In §III we describe consequences of moduli decay, namely, amount of entropy and gravitinos from them. Neutralino abundance from decaying gravitino is studied in $\S \mathrm{IV}$. Then we compare our yield with the constraints imposed by BBN in $\S \mathrm{V}$. Finally $\S \mathrm{VI}$ is devoted to conclusion and discussion.

\section{A SUCCESSFUL SCENARIO WITH SMALL HIERARCHY: OUR PROPOSAL}

In superstring theory and supergravity, there often exist scalar fields which have (almost) flat potential. Examples include moduli fields and the dilaton field in classical string vacua, 
whose vacuum expectation values (VEVs) determine the size and shape of compactified extra dimensions and the string coupling constant, respectively. They also include the field which is responsible for supersymmetry breaking in a typical gravity mediated supersymmetry breaking scenario (the Polonyi field). They typically have Planck-scale suppressed interaction, and the VEVs of the order of the Planck scale. We will generically call these fields the moduli fields.

It is expected that these moduli fields acquire non-flat potential and thus masses in some manner. Their masses depend on the mechanism how the moduli are stabilized. In a conventional view [12], the moduli fields acquire superpotential from some effect associated with supersymmetry breaking. In this case one naively expects that their masses are of the order of the gravitino mass, a typical mass scale of supersymmetry breaking in supergravity.

Recent development of flux compactification reveals the possibility that moduli yield superpotential when higher-form antisymmetric tensor fields acquire VEVs in compact directions [18]. The flux compactification fixes some of the moduli fields, but not all. In particular, in the type II superstring the overall modulus whose VEV determines the size of the compactified manifold is not stabilized in this way. These remaining moduli may acquire potential by other non-perturbative effects, and we may expect that their masses are related to the gravitino mass scale. $[19,20]$

Thus the naive expectation of the gravity mediation of supersymmetry breaking is that the masses of the moduli fields and sparticles in the MSSM sector are similar, and is of the order of the gravitino mass. Since the sparticle masses are expected to be in (sub-) TeV range, the naive guess leads that the gravitino mass as well as the moduli masses are also around this range. In fact, the cosmological moduli problem and the gravitino problem are consequences of this naive expectation.

A closer look at these masses, however, reveals possible deviation of the mass scales in one or two orders of magnitude. In the original gravity mediation model by Polonyi, the SUSY mass scale is given by hand, and the mass of the Polonyi field is indeed comparable to the gravitino mass. In more realistic models, the potential of the moduli fields may be generated dynamically. A typical example of this comes from gauge dynamics such as gaugino condensation [21]. In this scheme, the hierarchically small SUSY breaking scale is achieved by the dimensional transmutation with some numerical coefficient of order 10-100. At the same time, the moduli mass which is determined by the second derivative of the scalar potential can be larger by the same amount than the gravitino mass which is naively expected. In fact, this is the case for the well-known racetrack scenario [22] where multiple gaugino condensates take place to obtain a non-trivial minimum of the scalar potential. Similar observation has been made in other scenarios, including non-perturbative correction to the Kähler potential of the dilaton field [23]. For explicit demonstration, see [24]. The moduli masses can also be heavy in some scenarios of flux compactification [20].

This consideration sets a small hierarchy between the moduli masses and the gravitino mass. Another small hierarchy between the gravitino mass and the sparticle masses in the MSSM can be realized when the field responsible for the SUSY breaking is somewhat sequestered from the MSSM sector [25-27]. It is interesting to note that the coupling of the moduli fields to the MSSM sector does not violate this property of sequestering, because the moduli fields are heavy so that their auxiliary fields have SUSY breaking VEVs suppressed by the moduli masses relative to the gravitino mass. Then the softly SUSY breaking masses 
for sparticles are a combination of the contribution from the moduli SUSY breaking and that from the anomaly mediation. [26,28]

The small mass hierarchy among the moduli, gravitino and sparticle masses given above yields a successful scenario without the difficulties of the gravitino as well as the neutralino abundance. Here we would like to sketch our proposal. From now on, we assume, for simplicity, that there is only one modulus field which is relevant in our discussion.

The heavy modulus field is expected to obey damped coherent oscillation with the initial amplitude of order the Planck scale. The modulus oscillation dominates the energy density of the universe until it decays to reheat the universe. In the present case, since the modulus is rather heavy, its lifetime can be much shorter than 1 sec. The entropy production at the reheating dilutes the primordial abundances of the gravitinos. Non-negligible amount of gravitinos are, however, produced by the modulus decay, on which the constraint from BBN will be imposed.

The sparticles in the MSSM sector are also diluted by the modulus decay, if the decay products do not contain the sparticles and the thermal bath after the reheating is not hot enough to regenerate the sparticles by scattering processes. We will check that the former is indeed the case when the modulus coupling to the MSSM sector is of certain type, and the latter will be guaranteed if the reheating temperature is low enough. In this case, the neutralinos which remain until today come from the gravitino decay. The main assertion of this paper is that in some regions of the parameter space the neutralinos can be abundant to constitute the dark matter of the universe, whereas the gravitino abundance satisfies the constraint from the BBN.

The rest of the paper is devoted to describe the details of our proposal given above.

\section{MODULI DECAY: ENTROPY PRODUCTION AND GRAVITINO PRODUCTION}

The modulus field is typically displaced from the true minimum during the inflation, which starts damped coherent oscillation when the expansion rate of the universe decreases to the modulus mass. Unless the initial amplitude of the modulus field $\phi_{i}$ is very small compared to the Planck scale, the energy density of the modulus field in the form of the coherent oscillation will soon dominate the universe. The modulus eventually decays. The decay depends on how it couples to other particles [29]. Here we assume that it decays mainly to the ordinary particles in the MSSM and thus the universe becomes radiation dominated again. This is especially the case when the modulus coupling to the gauge bosons and gauginos through gauge kinetic function gives the largest contribution to the decay, where the modulus decays dominantly to a gauge boson pair while the decay to a gaugino pair suffers chirality suppression. We write the total decay width of the modulus field

$$
\Gamma_{\text {tot }}=N \frac{m_{\phi}^{3}}{M_{G}^{2}},
$$

where $m_{\phi}$ is the mass of the modulus field, $M_{G}=2.4 \times 10^{18} \mathrm{GeV}$ represents the reduced Planck scale, and $N$ parameterizes our ignorance of the interaction of the modulus. It is natural to expect that $N \sim 10^{-2}-1$. Given the above expression, the reheat temperature after the modulus decay $T_{R}$ is calculated to be 


$$
\begin{aligned}
T_{R} & =\left(\frac{90}{\pi^{2} g_{*}}\right)^{1 / 4} \sqrt{\Gamma_{\mathrm{tot}} M_{G}}=\left(\frac{90 N^{2}}{\pi^{2} g_{*}}\right)^{1 / 4} m_{\phi}^{3 / 2} M_{G}^{-1 / 2} \\
& \approx 11 N^{1 / 2}\left(\frac{g_{*}}{10^{2}}\right)^{-1 / 4}\left(\frac{m_{\phi}}{10^{4} \mathrm{TeV}}\right)^{3 / 2} \mathrm{GeV}
\end{aligned}
$$

where $g_{*}$ counts the effective relativistic degrees of freedom with $g_{*}=228.75$ for the MSSM particles.

Entropy production is also computed in a straightforward way. The entropy increase factor is found to be [13]

$$
\Delta=\sqrt{\frac{2}{9 N}} \frac{M_{G}}{m_{\phi}}\left(\frac{\phi_{i}}{M_{G}}\right)^{2}
$$

Thus, as far as the modulus mass is much lower than the Planck scale and the initial amplitude of the modulus oscillation $\phi_{i}$ is not extremely small, the entropy increase factor is much larger than unity, which we assume to be the case. The gravitino abundance as well as the sparticle abundances before the reheating will be diluted.

Let us now discuss the production of the gravitinos by the modulus decay. It has been pointed out in [30] that the modulus field generically decays to a pair of the gravitinos via the following terms in the supergravity Lagrangian:

$$
\mathcal{L}_{\text {int }}=-e^{K / 2} W \bar{\psi}_{\mu} \sigma^{\mu \nu} \bar{\psi}_{\nu}+H . c .
$$

where $\psi_{\mu}$ represents the gravitino field, $K$ is the Kähler potential, and $W$ is the superpotential. In the above expression, we have the reduced Planck scale $M_{G}$ to be unity. Since $K$ and $W$ have the moduli dependence, the above term in the Lagrangian gives the coupling of the modulus to the gravitino pair. Explicitly this is given by

$$
\left(e^{K / 2} W\right)_{\phi}=\frac{K_{\phi}}{2} e^{K / 2} W+e^{K / 2} W_{\phi},
$$

where the suffix $\phi$ here stands for the derivative with respect to $\phi$. Now the auxiliary component of the modulus field is written

$$
\bar{F}^{\bar{\phi}}=-e^{K}\left(K^{-1}\right)^{\bar{\phi} \phi}\left(W_{\phi}+K_{\phi} W\right) .
$$

As we assume that the modulus field is not a dominant source of SUSY breaking, we can approximately equate $W_{\phi}=-K_{\phi} W$. Using this, (6) can be rewritten as follows:

$$
\left(e^{K / 2} W\right)_{\phi}=-\frac{1}{2} K_{\phi} e^{K / 2} W=-\frac{1}{2} K_{\phi} m_{3 / 2}
$$

Thus the partial decay width to the gravitino pair becomes of the form

$$
\Gamma\left(\phi \rightarrow 2 \psi_{\mu}\right)=C \frac{m_{3 / 2}^{2}}{M_{G}^{2}} m_{\phi}=C \frac{m_{\phi}^{3}}{M_{G}^{2}}\left(\frac{m_{3 / 2}}{m_{\phi}}\right)^{2},
$$

where we have recovered the reduced Planck scale $M_{G}$ explicitly. In this expression, we have introduced a parameter $C \sim 1 / 32 \pi\left|K_{\phi} / M_{G}\right|^{2}$, where a possible numerical factor as well as 
the phase space factor is ignored. We expect that $C \sim 10^{-2}-10^{-1}$ would be natural, but a smaller $C$ may also be possible.

It has recently been shown that a more effective decay channel to the gravitino and the modulino, the fermionic component of the modulus supermultiplet, is kinematically allowed if a certain coupling of the modulus to the field responsible to the SUSY breaking is large enough [13]. Whether this channel is allowed or not is, however, quite model dependent. Here we conservatively consider the former decay mode to the gravitino pair, which will also provide a preferred result as we will see shortly.

Gravitinos are also produced by scattering processes in the thermal bath after the reheating epoch. The production rate is proportional to the reheat temperature and the ratio of the number density of thermally produced gravitinos to the entropy density after modulus decay reads $[31,32,3]$,

$$
\begin{aligned}
Y_{3 / 2}^{\mathrm{th}} & \simeq 1.6 \times 10^{-12}\left(\frac{T_{R}}{10^{10} \mathrm{GeV}}\right) \\
& =2 \times 10^{-9}\left(\frac{N}{10}\right)^{1 / 2}\left(\frac{g_{*}}{10^{2}}\right)^{-1 / 4}\left(\frac{m_{\phi}}{10^{5} \mathrm{TeV}}\right)^{3 / 2} .
\end{aligned}
$$

With the reheat temperature much below $1 \mathrm{TeV}$ which we are interested in, the gravitino production by the scattering processes is negligibly small.

As was shown in [13], the gravitino problem will be solved in this scenario. The primordial abundance of the gravitino generated before the reheating due to the modulus decay will be diluted to a negligible level. Although the gravitinos are produced by the modulus decay, the abundance was shown to be small enough to survive the constraints from the big-bang nucleosynthesis in an appropriate region of the parameter space. Here, we consider the decay mode of the modulus to the gravitino pair. Then the allowed region in the parameter space will become larger than that of Ref. [13].

More explicitly, let us estimate the gravitino abundance produced by the modulus decay. Here we approximate the decay of the modulus field as an instantaneous decay. The energy density of the modulus field is equal to that of the radiation at the reheating, i.e. at the modulus decay:

$$
\rho_{R}=m_{\phi} n_{\phi}
$$

where $n_{\phi}$ is the number density of the modulus. The gravitino number density at the modulus decay $n_{3 / 2}$ can also be easily estimated in the approximation

$$
n_{3 / 2}=2 B n_{\phi}
$$

where $B$ represents the branching ratio of the modulus decay into the gravitino pair

$$
B \equiv \operatorname{Br}\left(\phi \rightarrow 2 \psi_{\mu}\right)=\frac{C}{N}\left(\frac{m_{3 / 2}}{m_{\phi}}\right)^{2} .
$$

The gravitino abundance in the form of yield $Y_{3 / 2}$, the ratio of the number density relative to the entropy density, is given by

$$
Y_{3 / 2}=\frac{3}{4} \frac{n_{3 / 2} T_{R}}{\rho_{R}}=\frac{3}{2} B \frac{T_{R}}{m_{\phi}}=1.8 \times 10^{-10} C N^{-1 / 2}\left(\frac{g_{*}}{10^{2}}\right)^{-1 / 4}\left(\frac{m_{3 / 2}}{10^{2} \mathrm{TeV}}\right)^{2}\left(\frac{m_{\phi}}{10^{5} \mathrm{TeV}}\right)^{-3 / 2} .
$$




\section{NEUTRALINO ABUNDANCE}

In this section, we would like to discuss the relic abundance of the neutralinos. Here we assume that the R-parity is conserved and the lightest neutralino becomes the lightest superparticle, and thus it is a candidate for dark matter of the universe.

The entropy production at the modulus decay completely dilutes the primordial abundance of the neutralinos and other superparticles. Thus in our scenario the neutralinos which remain today should be produced at and after the reheating due to the modulus decay. There are three possible sources of the neutralinos:

- Neutralinos produced by gravitino decay

- Neutralinos produced by modulus decay

- Neutralinos produced in the thermal bath during and after the reheating

We now show that the first contribution can dominate over the other two.

As we discussed earlier, the universe is reheated at the modulus decay. If the reheat temperature were too high, the neutralinos would again be in the thermal equilibrium. In this case the standard computation of the neutralino relic abundance would apply, resulting in the potential problem of the too large relic abundance unless some enhancement mechanism of their annihilation is at work. This would be the case if the reheat temperature exceeded $m_{\chi} / 20 \sim m_{\chi} / 25$, where $m_{\chi}$ is the mass of the lightest neutralino. On the other hand, if the reheat temperature is fairly low, the regeneration of the neutralinos in the thermal bath is negligibly small. Therefore we will consider the case where $T_{R}<m_{\chi} / 30$ in the following. Note that this condition is not the constraint, but the requisition in the current interest.

Let us next discuss the neutralinos produced by the gravitino decay. The point here is that the gravitino decays eventually to the neutralino LSP under the R-parity conservation, and the number of the neutralinos is essentially the same as that of the gravitinos. Thus the yield of the neutralino LSPs is evaluated as

$$
Y_{\mathrm{LSP}}=Y_{3 / 2}=1.8 \times 10^{-10} C N^{-1 / 2}\left(\frac{g_{*}}{10^{2}}\right)^{-1 / 4}\left(\frac{m_{3 / 2}}{10^{2} \mathrm{TeV}}\right)^{2}\left(\frac{m_{\phi}}{10^{5} \mathrm{TeV}}\right)^{-3 / 2}
$$

This is a constant of time, if there is no other process which will change the number of the neutralinos nor the total entropy. We would like to emphasize that the neutralino abundance obtained in this mechanism is insensitive to the details of the superparticle mass spectrum, unlike the standard thermal abundance because $Y_{\text {LSP }}$ is directly related with only $Y_{3 / 2}$. Annihilation of the neutralinos, if it took place, would change it. We will come back to this issue later on. On the other hand, using the density parameter of LSP, $Y_{\mathrm{LSP}}$ is also represented by

$$
Y_{\mathrm{LSP}}=3.65 \times 10^{-11} \Omega_{\mathrm{LSP}} h^{2}\left(\frac{m_{\mathrm{LSP}}}{100 \mathrm{GeV}}\right)^{-1}\left(\frac{T_{0}}{2.725 \mathrm{~K}}\right)^{-3},
$$

where we adopted the present photon temperature $T_{0}=2.725 \pm 0.002 \mathrm{~K}[33]$.

Neutralinos may also be produced by the modulus decay. Let us again consider the case where the modulus couples to the bilinear of the supersymmetric form of the gauge potential. 
In this case, the decay to the gaugino pair is known to receive chirality suppression, and thus the branching ratio is roughly estimated as

$$
\operatorname{Br}(\phi \rightarrow 2 \tilde{\mathrm{g}}) \sim\left(\frac{m_{\tilde{g}}}{m_{\phi}}\right)^{2}
$$

where $\tilde{g}$ symbolically denotes a gaugino. This should be compared with the branching ratio to the gravitino pair as

$$
\frac{\operatorname{Br}(\phi \rightarrow 2 \tilde{\mathrm{g}})}{\operatorname{Br}\left(\phi \rightarrow 2 \psi_{\mu}\right)} \sim \frac{N}{C}\left(\frac{m_{\tilde{g}}}{m_{3 / 2}}\right)^{2}
$$

Note that we are considering the case where $\left(m_{\tilde{g}} / m_{3 / 2}\right)^{2} \sim 10^{-4}-10^{-3}$. Thus the contribution to the neutralino abundance from the direct modulus decay is sub-dominant, as far as the ratio $N / C$ does not exceed $\sim \mathcal{O}\left(10^{4}\right)$.

The addition of the coupling of the modulus to two chiral multiplets (e.g. quarks and their superpartners) does not change the conclusion given above, because the decay to a squark pair, for instance, is suppressed by the fourth power of the mass ratio $m_{\tilde{q}} / m_{3 / 2}$, unlike the second power for the case of the gaugino pair [29]. Thus it is negligible, provided that the coupling of the modulus to the gauginos is not small. In the following, we shall not consider the direct modulus decay to superparticles in the MSSM.

In the following we assume that the pair annihilation of the neutralinos after the production by the gravitino decay does not take place. Whether the annihilation is effective or not can be seen by comparing the annihilation rate to the Hubble expansion rate of the universe when the neutralinos are produced by the gravitino decay. Although the annihilation cross section of the neutralino $\sigma_{\text {ann }}$ is a function of the superparticle mass spectrum, it is generically bounded as

$$
\sigma_{\text {ann }} \lesssim \frac{\alpha_{1}}{m_{\chi}^{2}}
$$

with the coupling $\alpha_{1} \sim 10^{-2}$. Then, the ratio of the annihilation rate $\Gamma_{\mathrm{ann}}=n_{\mathrm{LSP}} \sigma_{\mathrm{ann}}$, to the Hubble expansion rate $H \sim T^{2} / M_{\mathrm{G}}$ is bounded as

$$
\frac{\Gamma_{\text {ann }}}{H}<\sigma_{\text {ann }} y_{3 / 2}^{\max } s \frac{M_{\mathrm{G}}}{T^{2}}=10^{-4}\left(\frac{m_{\chi}}{100 \mathrm{GeV}}\right)^{-3}\left(\frac{T}{\mathrm{MeV}}\right) .
$$

Therefore, we find $\Gamma_{\mathrm{ann}} / H<1$ at the temperature of gravitino decay.

\section{RESULTS}

We are ready to present our numerical results now. We first discuss constraints we impose on the parameter space. Constraints on the reheat temperature after moduli decay are that it should be 1) higher than the lower bound required by the successful BBN, $T_{R}>1.2$ 
$\mathrm{MeV}[16],{ }^{1}$ but 2) lower than the temperature above which the neutralinos are thermalized. We set the latter condition as $T_{R}<m_{\mathrm{LSP}} / 30$.

We show the constraint on gravitino decay from the BBN in Fig. 1. The upper bound on the yield of the gravitino $n_{3 / 2} / s$ is drawn as a function of the gravitino mass. Effects of hadronic shower are taken into account [3]. Here we take the hadronic branching ratio to be 0.8 because such a heavy gravitino can decay into all of the gauge boson and the gaugino pairs and the chiral fermion and its scalar partner pairs. ${ }^{2}$ For gravitino mass lighter than $10 \mathrm{TeV}$, the constraint comes from the overproduction of ${ }^{6} \mathrm{Li}$ and $\mathrm{D}$ abundances. On the other hand, the case with heavier mass is constrained by the ${ }^{4} \mathrm{He}$ overproduction. This is because the inter-converting reaction between the neutron and the proton becomes effective, which is induced by the pions and nucleons emitted by the hadronic decays of the gravitinos. Here we adopted the observational value of the ${ }^{4}$ He mass fraction $Y_{p}(I T)=0.242 \pm 0.002$ (stat) $\left( \pm 0.005\right.$ (syst)) by Izotov and Thuan (2004) [34]. ${ }^{3}$ In the same figure, the dotted strip indicates the region where the LSP abundance from the gravitino decay corresponds to the desired value $\Omega_{\mathrm{LSP}} h^{2}=0.0945-0.1287$ (95\% C.L.) in Eq. (16). Here we have assumed the LSP mass, $m_{\mathrm{LSP}}$, to be $100 \mathrm{GeV}$. One can easily scale the strip for other values of $m_{\mathrm{LSP}}$ by using Eq. (16).

By equating Eq. (15) with Eq. (16), we see that the allowed value of $m_{\phi}$ is proportional to $C^{2 / 3}$ for a fixed value of $\Omega_{\mathrm{LSP}} h^{2}$. In Fig. 2, we plot the allowed regions for suitable observational value of $\Omega_{\mathrm{LSP}} h^{2}=0.0945-0.1287$ (95\% C.L.) in the $C-m_{\phi}$ plane. We plotted the strips for $m_{3 / 2}=50,10^{2}, 5 \times 10^{2}$, and $10^{3} \mathrm{TeV}$, respectively. We considered only two conditions that $T_{R}<m_{\mathrm{LSP}} / 30$ and $m_{\phi}>2 m_{3 / 2}$. Here we took $N=1$, and $m_{\mathrm{LSP}}=100 \mathrm{GeV}$. As a result we find that $C$ should lie approximately in the range of $10^{-4} \lesssim C \lesssim 10^{-1}$. Note that the theoretically natural value of $C \sim 10^{-2}-10^{-1}$ is completely included in this range.

We can now identify regions of the parameter space where the LSP neutralino from the

\footnotetext{
${ }^{1}$ Here we adopted a milder lower bound on the reheat temperature when we assume that the modulus decays only into photons. On the other hand, if we consider the case that the decaying modulus also emits hadrons, the lower bound becomes higher $T_{R} \gtrsim 4-5 \mathrm{MeV}$ [16]. However, this difference does not change our results at all for the current purpose.

${ }^{2}$ According to the treatments in Ref. [3], we could not include all of the hadronic decay modes into the BBN computation at one time. Therefore, here we consider only the most dominant mode into the hadrons, i.e., the gluon-gluino pairs. Then the branching ratio into the gluon-gluino pairs is approximately 0.5. Because we adopted this value for the BBN computation, the obtained bound on $y_{3 / 2}=n_{3 / 2} / s$ in Fig. 1 might be milder by $\sim 30 \%$ approximately. Note, however, that this difference of the hadronic branching ratio does not change the constraints on the model parameters so much nor does our results at all. For the more accurate computations, in which we include all of the hadronic decay modes at one time, we would like to discuss them in separate issues.

${ }^{3}$ Recently much milder value of the observational ${ }^{4} \mathrm{He}$ mass fraction was reported by Olive and Skillman, $Y_{p}(O S)=0.249 \pm 0.009$ [35]. Because their method is not the standard way to obtain the temperature and the electron density, we adopt it as a reference value here. The constraint which comes from $Y_{p}(O S)$ is denoted by the thin solid line in the figures hereafter.
} 
gravitino decay becomes the dark matter of the universe. Fig. 3 depicts various constraints on the $m_{3 / 2}-m_{\phi}$ plane, (i) BBN constraints (thick and thin solid lines), (ii) $T_{R}<m_{\mathrm{LSP}} / 30$ (long dashed line), (iii) $T_{R}>1.2 \mathrm{MeV}$ (dashed line), and (iv) $m_{\phi}>2 m_{3 / 2}$ (dot-dashed line) in the case $C=10^{-2}$ and $N=1$. Also depicted there are two strips where neutralino abundance from decaying gravitinos is in accord with the recently observed value $\Omega_{\mathrm{LSP}} h^{2}=$ $0.0945-0.1287\left(95 \%\right.$ C.L.) with $m_{\mathrm{LSP}}=100 \mathrm{GeV}$. The upper strip corresponds to the case gravitinos are thermally produced after modulus decay. However, it is entirely covered by the region with $T_{R}>m_{\mathrm{LSP}} / 30$ where the reheat temperature is so high that LSPs are thermally populated after modulus decay. In this case dark matter LSPs tend to be overpopulated unless some enhancement mechanism of annihilation is operative. This long dashed line has a scaling law for the other values of $m_{\mathrm{LSP}}$ as $m_{\phi} \propto m_{\mathrm{LSP}}^{2 / 3}$. The lower strip, on the other hand, represents the case gravitinos are produced directly by modulus decay. There is a region on this strip where all the constraints are satisfied simultaneously with $m_{3 / 2} \simeq 55-100 \mathrm{TeV}$ and $m_{\phi} \simeq(2-4) \times 10^{3} \mathrm{TeV}$. As is seen in the figure it is highly nontrivial that such a region really exists. Since $N$ appears only in the form $N^{1 / 2} m_{\phi} 3 / 2$ in all of the relevant expressions in Eqs. (3), (10) and (14), the constraints for $N$ other than $N=1$ can easily be read off by replacing $m_{\phi}$ in the vertical axis by $N^{1 / 3} m_{\phi}$. Concerning this lower strip, there is a scaling relation $m_{\phi} \propto m_{\mathrm{LSP}}^{2 / 3}$. Thus, for the other values of $m_{\mathrm{LSP}}$ we can easily see how the results change by using this relation.

Fig. 4 depicts how the results change as we take different values of $C$ which parameterizes the branching ratio of the modulus decay into the gravitino pair. We plot the cases (a) $C=10^{-4}$, (b) $C=10^{-3}$, (c) $C=10^{-2}$, and (d) $C=10^{-1}$, respectively. From this panel, we can find that wide ranges of $m_{\phi}$ are allowed $\left(\sim \mathcal{O}\left(10^{2}\right)-\mathcal{O}\left(10^{3}\right) \mathrm{TeV}\right)$ when $C$ is changed for $\sim \mathcal{O}\left(10^{-4}\right)-\mathcal{O}\left(10^{-1}\right)$. Here, note that the intersection between the trip and the boundary of the BBN constraint gives a same value of $m_{\phi}(\sim 55 \mathrm{TeV})$ for any values of $C$.

In Fig. 5 we depict the domain in the $m_{3 / 2}-m_{\phi}$ plane where $\Omega_{\mathrm{LSP}} h^{2}$ can take the observed value with some value of $C$ in the range $C \sim 10^{-4}-10^{-1}$. The vertical thick (thin) solid line represents the BBN constraint, which comes from the observational ${ }^{4} \mathrm{He}$ mass fraction by Izotov and Thuan $Y_{p}(I T)$ ( Olive and Skillman $Y_{p}(O S)$ ). The long dashed line represents the boundary of $T_{R}<m_{\mathrm{LSP}} / 30$. The dot-dashed line denotes the boundary of $m_{\phi}>2 m_{3 / 2}$.

As is seen there, we have a fairly large allowed region for $m_{3 / 2} \simeq 55-2 \times 10^{3} \mathrm{TeV}$ and $m_{\phi} \simeq 10^{2}-4 \times 10^{3} \mathrm{TeV}$, with a small hierarchy between $m_{\phi}$ and $m_{3 / 2}$.

For different values of $m_{\mathrm{LSP}}$, because the boundary of $T_{R}<m_{\mathrm{LSP}} / 30$ has a scaling law

as $m_{\phi} \propto m_{\mathrm{LSP}}^{2 / 3}$, we can rescale the long dashed line by using this relation. In addition, about the modification of the vertical thick and thin lines for the other values of $m_{\mathrm{LSP}}$, we can read off their values of $m_{3 / 2}$ as the intersections between the trip and the boundaries of the BBN constraint in Fig. 1 or Fig. 3. The long dashed line for $N$ other than $N=1$ can easily be read off by replacing $m_{\phi}$ in the vertical axis by $N^{1 / 3} m_{\phi}$.

\section{DISCUSSION}

We have shown that the neutralino dark mater whose abundance agrees with the WMAP constraint can be non-thermally produced by the decay of heavy gravitino with the mass of $m_{3 / 2} \sim 55-100 \mathrm{TeV}$ in the early universe. Such gravitinos are produced only by the 
decaying moduli with the late-time large entropy production, which completely dilutes both the thermal relics of LSPs, and primordial gravitinos produced by the reheating process after the primordial inflation. In this case, we found that the required region of the moduli mass is $m_{\phi} \simeq 10^{2}-4 \times 10^{3} \mathrm{TeV}$. This scenario can solve the overproduction problem of LSPs in the supersymmetric cosmology.

Note that although the LSP is still the best candidate for cold dark matter in particle physics, the recent measurement of the abundance by the WMAP restricts the preferred regions of the SUSY parameter space. The old and naive argument that the dark matter abundance is of the order of the critical density of the universe, which allows the wide regions of the parameter space, does no longer apply today because its abundance has been measured to be only one quarter of the critical density, which turned out to be significantly smaller than we expected in the standard calculation. As mentioned in the introduction, attempts do exist to explain this smallness within the standard thermal history, but this in turn requires us to adopt some enhancement mechanism of pair annihilation such as coannihilation or resonance enhancement etc (For the details see, e.g. recent works [36,37]). Our scenario is in a sense more economical than these mechanisms because we can account for the desired abundance of the neutralino dark matter as these particles are produced from modulus decay which also produces large entropy to solve the new gravitino problem at the same time.

It is interesting to point out an alternative application of the production mechanism described in this paper. The neutralino production by gravitino decay can allow the neutralino dark matter with large fraction of the Wino and Higgsino components: its thermal abundance would be too small to constitute the dark matter of the universe. In this case, the cross section of the neutralino-nuclei scattering and the neutralino-neutralino pair annihilation can become larger. Thus, in the direct searches such as CDMS [38], EDELWEISS [39], and DAMA [40], we expect a higher detection rate than that of pure Bino LSP. In the indirect searches, we can also expect higher detectability of the signal of photons, neutrinos, electrons and their radio emission through the neutralino-neutralino pair annihilation in the galactic center in the Milky Way, the center of the other galaxies, and the clusters of galaxies in the future experiments such as GLAST [41] and MAGIC [42] for gamma-rays, and Hyper-Kamiokande [43] and TITAND [44] for neutrinos, and so on.

Finally we would like to emphasize that in this scenario the large gravitino mass will make the superparticle mass spectrum very different from the conventional gravity mediated SUSY breaking, and we expect the admixture of gravity mediation and anomaly mediation [45-48], which will be testable in future collider experiments.

\section{ACKNOWLEDGMENTS}

This work was partially supported by the JSPS Grants-in Aid of the Ministry of Education, Science, Sports, and Culture of Japan No. 15-03605 (KK), No. 13640285 and No. 16340076 (JY), and No.12047201 (MY). K.K. was also supported by NSF grant AST 0307433. 


\section{REFERENCES}

[1] D. N. Spergel et al., Astrophys. J. Suppl. 148, 175 (2003) [arXiv:astro-ph/0302209].

[2] J. R. Ellis, K. A. Olive, Y. Santoso and V. C. Spanos, Phys. Lett. B 565, 176 (2003) [arXiv:hep-ph/0303043]. U. Chattopadhyay, A. Corsetti and P. Nath, Phys. Rev. D 68, 035005 (2003) [arXiv:hep-ph/0303201].

[3] M. Kawasaki, K. Kohri and T. Moroi, Phys. Lett. B 625, 7 (2005) [arXiv:astroph/0402490]; Phys. Rev. D 71, 083502 (2005) [arXiv:astro-ph/0408426].

[4] D. Lindley, Astrophys. J. 294 (1985) 1; M. Y. Khlopov and A. D. Linde, Phys. Lett. B 138, 265 (1984); F.Balestra et al., Sov. J. Nucl. Phys. 39, 626 (1984); M. Yu. Khlopov, Yu. L. Levitan, E. V. Sedelnikov and I. M. Sobol, Phys. Atom. Nucl. 571393 (1994); J. R. Ellis, J. E. Kim and D. V. Nanopoulos, Phys. Lett. B 145, 181 (1984). R. Juszkiewicz, J. Silk and A. Stebbins, Phys. Lett. B 158, 463 (1985); J. R. Ellis, D. V. Nanopoulos and S. Sarkar, Nucl. Phys. B 259 (1985) 175; J. Audouze, D. Lindley and J. Silk, Astrophys. J. 293, L53 (1985); D. Lindley, Phys. Lett. B 171 (1986) 235; M. Kawasaki and K. Sato, Phys. Lett. B 189, 23 (1987); R. J. Scherrer and M. S. Turner, Astrophys. J. 331 (1988) 19; J. R. Ellis et al., Nucl. Phys. B 373, 399 (1992).

[5] M. H. Reno and D. Seckel, Phys. Rev. D 37, 3441 (1988).

[6] S. Dimopoulos, R. Esmailzadeh, L. J. Hall and G. D. Starkman, Astrophys. J. 330, 545 (1988); Phys. Rev. Lett. 60, 7 (1988); Nucl. Phys. B 311, 699 (1989).

[7] M. Kawasaki and T. Moroi, Prog. Theor. Phys. 93, 879 (1995); Astrophys. J. 452, 506 (1995); E. Holtmann, M. Kawasaki, K. Kohri and T. Moroi, Phys. Rev. D 60, 023506 (1999).

[8] K. Jedamzik, Phys. Rev. Lett. 84, 3248 (2000); M. Kawasaki, K. Kohri and T. Moroi, Phys. Rev. D 63, 103502 (2001); R. H. Cyburt, J. R. Ellis, B. D. Fields and K. A. Olive, Phys. Rev. D 67, 103521 (2003).

[9] K. Kohri, Phys. Rev. D 64, 043515 (2001) [arXiv:astro-ph/0103411]. K. Kohri and J. Yokoyama, Phys. Rev. D 61, 023501 (2000) [arXiv:astro-ph/9908160].

[10] K. Sato, Mon. Not. Roy. Astron. Soc. 195, 467 (1981). A. H. Guth, Phys. Rev. D 23, 347 (1981).

[11] A.D. Linde, Particle Physics and Inflationary Cosmology (Harwood, Chur, Switzerland, 1990).

[12] G. D. Coughlan, W. Fischler, E. W. Kolb, S. Raby and G. G. Ross, Phys. Lett. B 131, 59 (1983). B. de Carlos, J. A. Casas, F. Quevedo and E. Roulet, Phys. Lett. B 318, 447 (1993) [arXiv:hep-ph/9308325]. T. Banks, D. B. Kaplan and A. E. Nelson, Phys. Rev. D 49, 779 (1994) [arXiv:hep-ph/9308292].

[13] K. Kohri, M. Yamaguchi and J. Yokoyama, Phys. Rev. D 70, 043522 (2004) [arXiv:hep$\mathrm{ph} / 0403043]$.

[14] T. Moroi, M. Yamaguchi and T. Yanagida, Phys. Lett. B 342, 105 (1995) [arXiv:hep$\mathrm{ph} / 9409367]$.

[15] L. Randall and S. Thomas, Nucl. Phys. B 449, 229 (1995) [arXiv:hep-ph/9407248].

[16] M. Kawasaki, K. Kohri and N. Sugiyama, Phys. Rev. Lett. 82, 4168 (1999) [arXiv:astroph/9811437]; Phys. Rev. D 62, 023506 (2000) [arXiv:astro-ph/0002127].

[17] S. Hannestad, Phys. Rev. D 70, 043506 (2004) [arXiv:astro-ph/0403291].

[18] S. B. Giddings, S. Kachru and J. Polchinski, Phys. Rev. D 66, 106006 (2002) [arXiv:hep- 
th/0105097]. S. Kachru, M. B. Schulz and S. Trivedi, JHEP 0310, 007 (2003) [arXiv:hepth/0201028].

[19] S. Kachru, R. Kallosh, A. Linde and S. P. Trivedi, Phys. Rev. D 68, 046005 (2003) [arXiv:hep-th/0301240].

[20] K. Choi, A. Falkowski, H. P. Nilles, M. Olechowski and S. Pokorski, JHEP 0411, 076 (2004) [arXiv:hep-th/0411066].

[21] H. P. Nilles, Phys. Lett. B 115, 193 (1982). S. Ferrara, L. Girardello and H. P. Nilles, Phys. Lett. B 125, 457 (1983). M. Dine, R. Rohm, N. Seiberg and E. Witten, Phys. Lett. B 156, 55 (1985).

[22] N. V. Krasnikov, Phys. Lett. B 193, 37 (1987). J. A. Casas, Z. Lalak, C. Munoz and G. G. Ross, Nucl. Phys. B 347, 243 (1990).

[23] T. Banks and M. Dine, Phys. Rev. D 50, 7454 (1994) [arXiv:hep-th/9406132].

[24] W. Buchmuller, K. Hamaguchi, O. Lebedev and M. Ratz, Nucl. Phys. B 699, 292 (2004) [arXiv:hep-th/0404168].

[25] K. Inoue, M. Kawasaki, M. Yamaguchi and T. Yanagida, Phys. Rev. D 45, 328 (1992).

[26] L. Randall and R. Sundrum, Nucl. Phys. B 557, 79 (1999) [arXiv:hep-th/9810155].

[27] M. A. Luty and R. Sundrum, Phys. Rev. D 62, 035008 (2000) [arXiv:hep-th/9910202].

[28] G. F. Giudice, M. A. Luty, H. Murayama and R. Rattazzi, JHEP 9812, 027 (1998) [arXiv:hep-ph/9810442].

[29] T. Moroi and L. Randall, Nucl. Phys. B 570, 455 (2000) [arXiv:hep-ph/9906527].

[30] M. Hashimoto, K. I. Izawa, M. Yamaguchi and T. Yanagida, Prog. Theor. Phys. 100, 395 (1998) [arXiv:hep-ph/9804411].

[31] T. Moroi, arXiv:hep-ph/9503210.

[32] M. Bolz, A. Brandenburg and W. Buchmuller, Nucl. Phys. B 606, 518 (2001).

[33] J.C. Mather et al., Astrophys. J. 512, 511 (1999).

[34] Y. I. Izotov and T. X. Thuan, Astrophys. J. 602, 200 (2004).

[35] K. A. Olive and E. D. Skillman, arXiv:astro-ph/0405588.

[36] J. R. Ellis, K. A. Olive, Y. Santoso and V. C. Spanos, Phys. Rev. D 70, 055005 (2004) [arXiv:hep-ph/0405110].

[37] L. Roszkowski and R. Ruiz de Austri, JHEP 0508, 080 (2005) [arXiv:hep-ph/0408227].

[38] CDMS Collaboration, http://cdms.berkeley.edu/

[39] EDELWEISS Collaboration, http://edelweiss.in2p3.fr/

[40] R. Bernabei et al., (DAMA Collaboration), Phys. Lett. B480, 23 (2000); Riv. NUovo Cimento, 26, (2003) 1 [astro-ph/0307403].

[41] GLAST Collaboration, http://www-glast.stanford.edu/

[42] The MAGIC Telescope Collaboration, http://wwwmagic.mppmu.mpg.de/

[43] K. Nakamura, Int. J. Mod. Phys. A 18 (2003) 4053.

[44] Y. Suzuki et al. [TITAND Working Group], arXiv:hep-ex/0110005.

[45] K. Choi, A. Falkowski, H. P. Nilles and M. Olechowski, Nucl. Phys. B 718, 113 (2005) [arXiv:hep-th/0503216].

[46] M. Endo, M. Yamaguchi and K. Yoshioka, Phys. Rev. D 72, 015004 (2005) [arXiv:hep$\mathrm{ph} / 0504036]$.

[47] K. Choi, K. S. Jeong and K. i. Okumura, arXiv:hep-ph/0504037.

[48] A. Falkowski, O. Lebedev and Y. Mambrini, arXiv:hep-ph/0507110. 


\section{FIGURES}

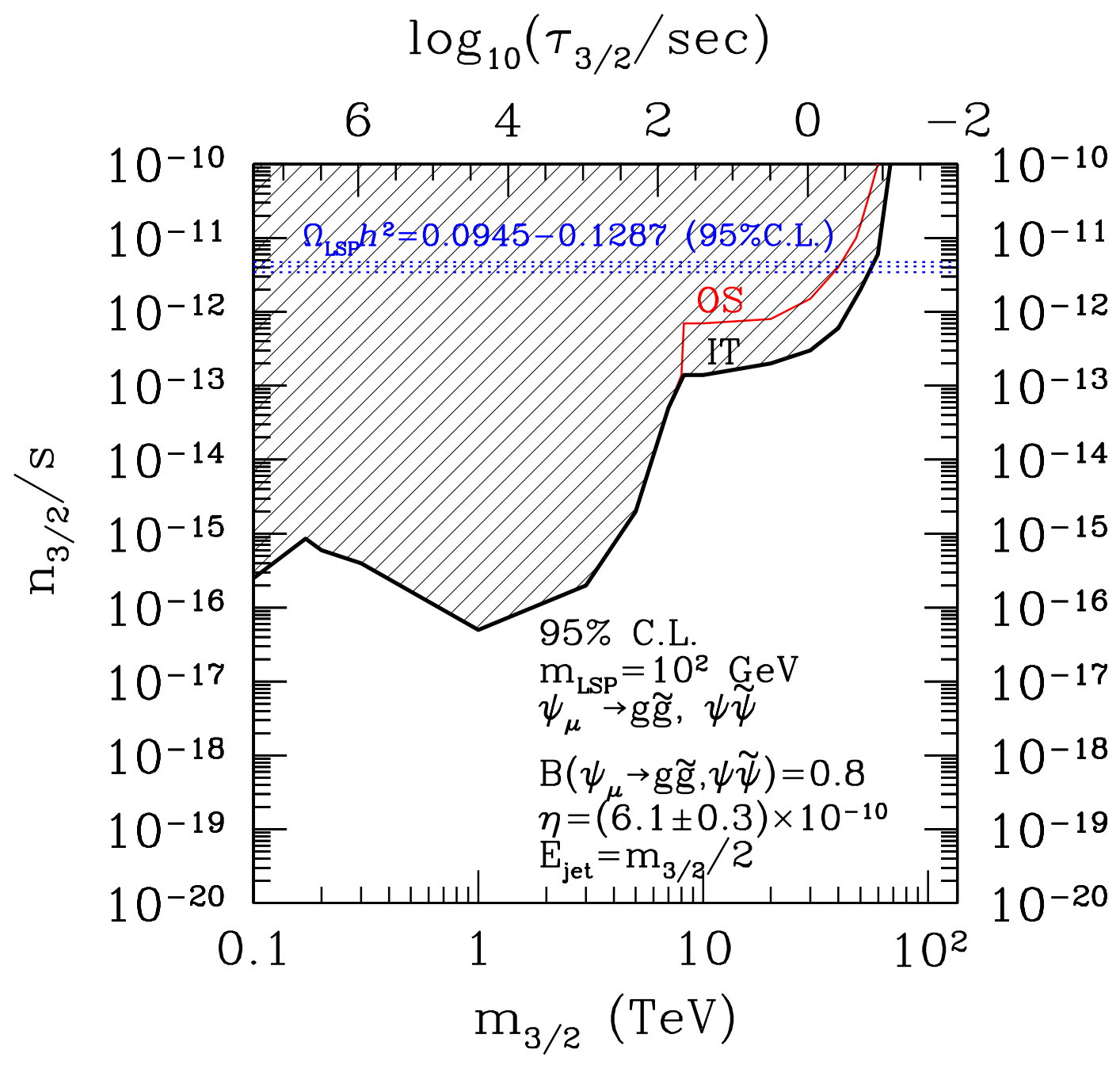

FIG. 1. Upper bound on the yield variable $y_{3 / 2}=n_{3 / 2} / s$. For heavy gravitinos with their masses of $m_{3 / 2}>10 \mathrm{TeV}$, the constraint mainly comes from the ${ }^{4} \mathrm{He}$ overproduction. The thick solid line, which is denoted by "IT", comes from the observational value of "He mass fraction by Izotov and Thuan (2003). The dotted strip indicates the region where the LSP abundance from the gravitino decay corresponds to the desired value $\Omega_{\mathrm{LSP}} h^{2}=0.0945-0.1287$ (95\% C.L.). The thin solid line, which is denoted by "OS", is the case that we adopted the most conservative observational value of $Y_{p}$ by Olive and Skillman (2004). Here we assumed that a gravitino can decay into all of the species of the chiral fermion and its scalar partner pairs $(\psi \tilde{\psi})$ and the gauge boson and the gaugino pairs $(g \tilde{g})$. 


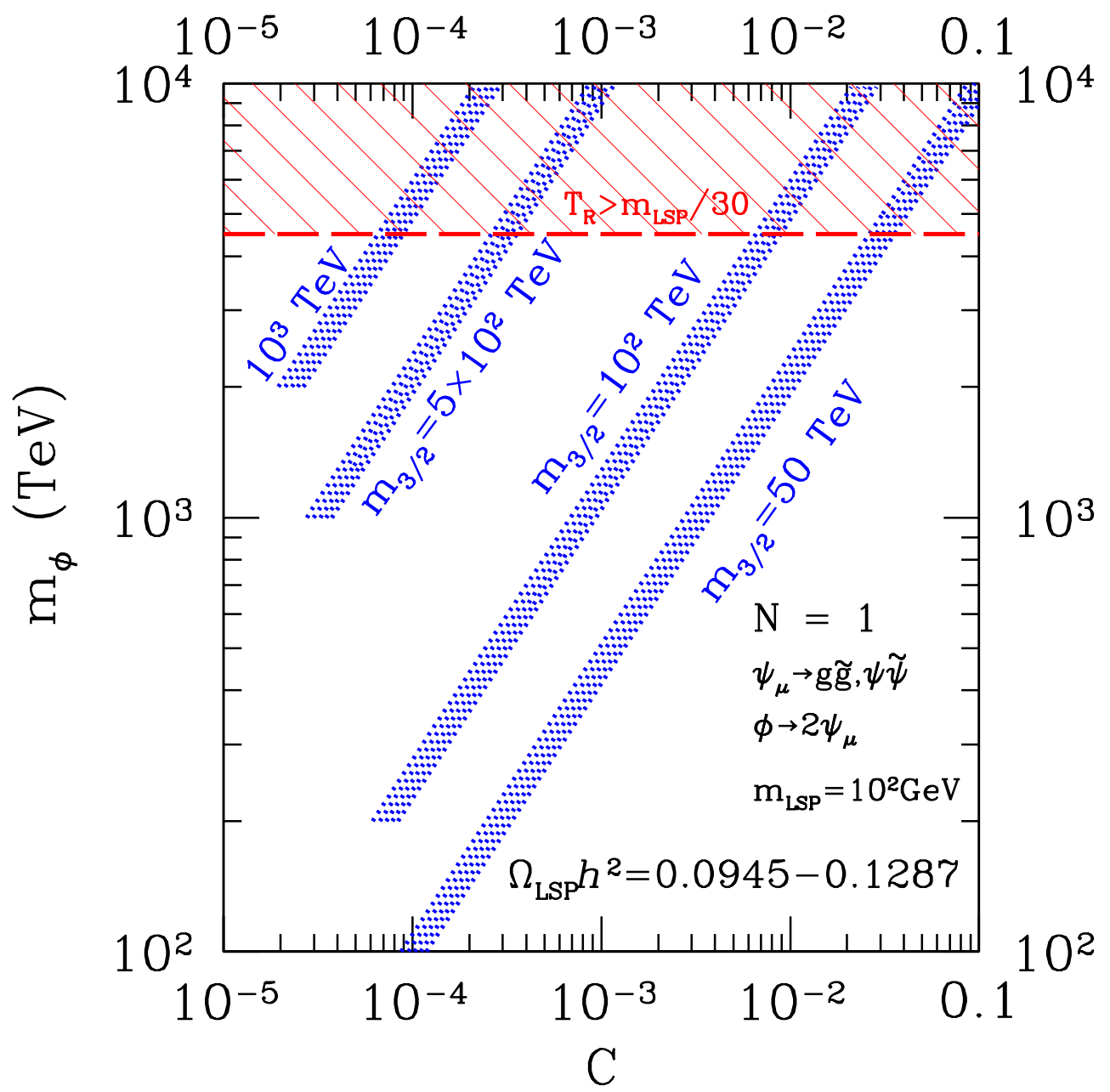

FIG. 2. Allowed regions for suitable observational value of $\Omega_{\mathrm{LSP}} h^{2}=0.0945-0.1287$ (95\% C.L.) in the $C-m_{\phi}$ plane. We plotted the strips for $m_{3 / 2}=50,10^{2}, 5 \times 10^{2}$, and $10^{3} \mathrm{TeV}$, respectively. We considered only two conditions that $T_{R}<m_{\mathrm{LSP}} / 30$ (below the long dashed line), and $m_{\phi}>2 m_{3 / 2}$. Here we took $N=1$, and $m_{\mathrm{LSP}}=100 \mathrm{GeV}$. 


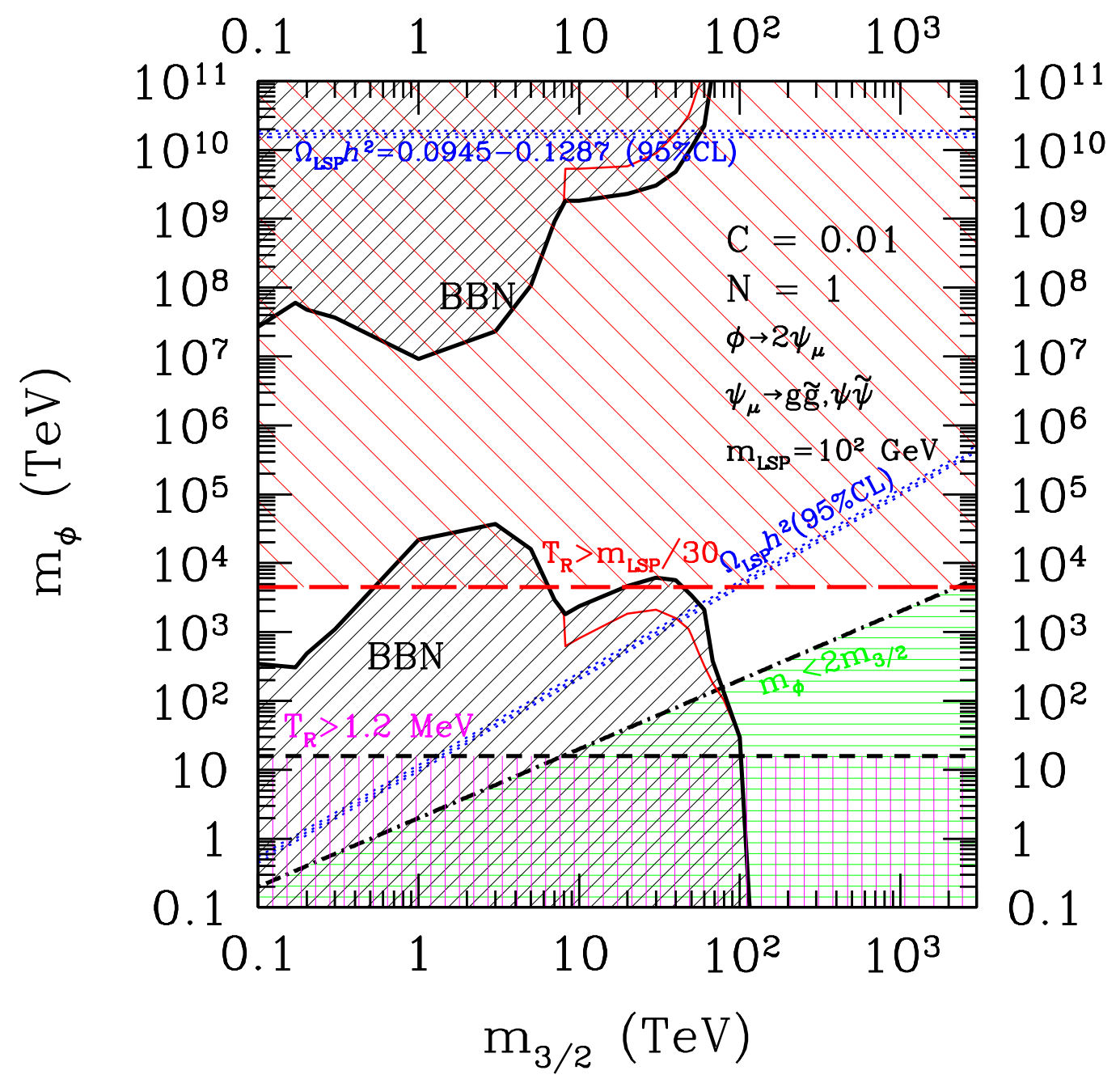

FIG. 3. Various constraints on the $m_{3 / 2}-m_{\phi}$ plane, (i) BBN constraints (thick and thin solid lines), (ii) $T_{R}<m_{\mathrm{LSP}} / 30$ (long dashed line), (iii) $T_{R}>1.2 \mathrm{MeV}$ (dashed line), and (iv) $m_{\phi}>2 m_{3 / 2}$ (dot-dashed line) in the case $C=10^{-2}$ and $N=1$. Also depicted there are two strips where neutralino abundance from decaying gravitinos is in accord with the recently observed value $\Omega_{\mathrm{LSP}} h^{2}=0.0945-0.1287$ (95\% C.L.) with $m_{\mathrm{LSP}}=100 \mathrm{GeV}$. There is a region on this strip where all the constraints are satisfied simultaneously with $m_{3 / 2} \simeq 55-100 \mathrm{TeV}$ and $m_{\phi} \simeq(2-4) \times 10^{3} \mathrm{TeV}$. Since $N$ appears only in the form $N^{1 / 2} m_{\phi}{ }^{3 / 2}$ in all of the relevant expressions in Eqs. (3), (10) and (14), the constraints for $N$ other than $N=1$ can easily be read off by replacing $m_{\phi}$ in the vertical axis by $N^{1 / 3} m_{\phi}$. 


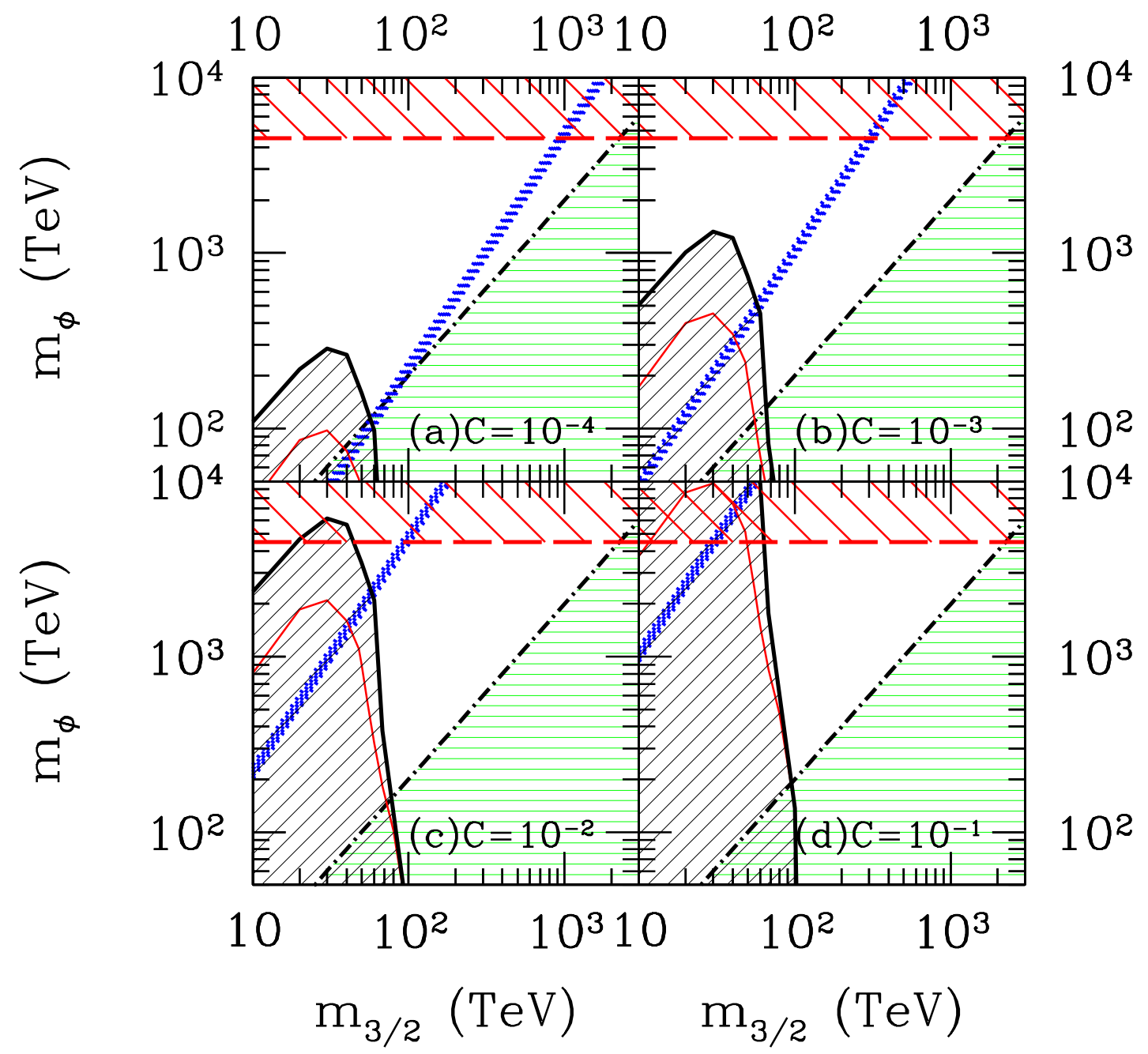

FIG. 4. How the results change as we take different values of $C$, which parameterizes the branching ratio of the modulus decay into the gravitino pair. We plot the cases (a) $C=10^{-4}$, (b) $C=10^{-3}$, (c) $C=10^{-2}$, and (d) $C=10^{-1}$, respectively. For the explanation of the various constraints, see the figure caption in Fig. 3. 


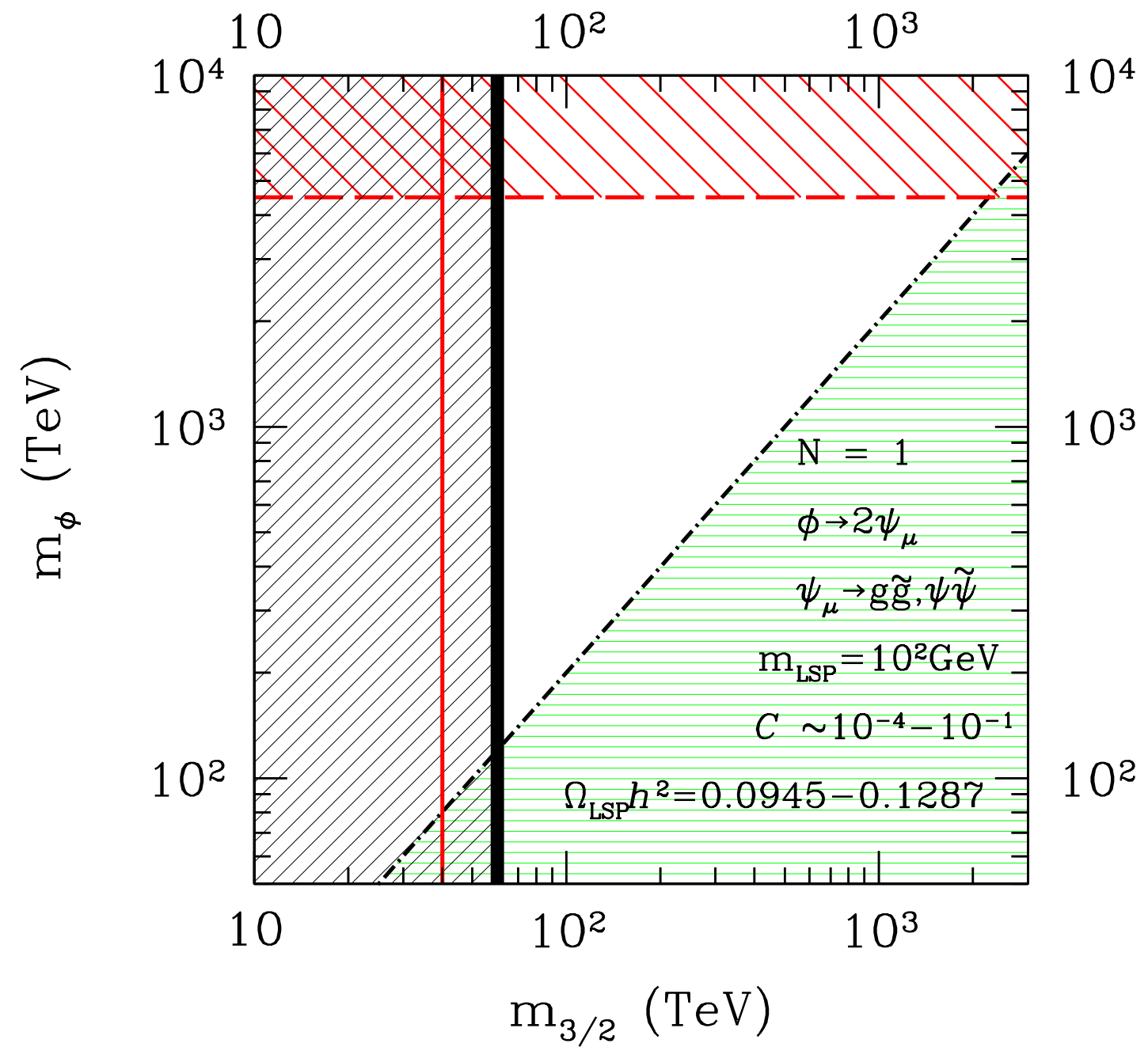

FIG. 5. Allowed region in the $m_{3 / 2}-m_{\phi}$ plane where $\Omega_{\mathrm{LSP}} h^{2}$ can take the observed value with $C \sim 10^{-4}-10^{-1}$. The vertical thick (thin) solid line represents the BBN constraint, which comes from the observational ${ }^{4} \mathrm{He}$ mass fraction by Izotov and Thuan $Y_{p}(I T)$ ( Olive and Skillman $\left.Y_{p}(O S)\right)$. The long dashed line represents the boundary of $T_{R}<m_{\mathrm{LSP}} / 30$. This line for $N$ other than $N=1$ can easily be read off by replacing $m_{\phi}$ in the vertical axis by $N^{1 / 3} m_{\phi}$. The dot-dashed line denotes the boundary of $m_{\phi}>2 m_{3 / 2}$. 